\title{
A Saúde e seus Determinantes Sociais
}

PAULO MARCHIORI BUSS

ALBERTO PELLEGRINI FILHO

\section{RESUMO}

Este artigo busca analisar as relações entre saúde e seus determinantes sociais, apresentando inicialmente o conceito de determinantes sociais de saúde (DSS) e uma breve evolução histórica dos diversos paradigmas explicativos do processo saúde/doença no âmbito das sociedades, desde meados do século XIX. Em seguida são discutidos os principais avanços e desafios no estudo dos DSS, com ênfase em novos enfoques e marcos de referência explicativos das relações ente os diversos níveis de DSS e a situação de saúde. Com base nesses estudos e marcos explicativos, discutese, em seguida, uma série de possibilidades de intervenções de políticas e programas voltados para o combate às iniqüidades de saúde geradas pelos DSS. Finalmente, são apresentados os objetivos, linhas de atuação e principais atividades da Comissão Nacional sobre Determinantes Sociais da Saúde, criada em março de 2006, com o objetivo de promover estudos sobre os DSS, recomendar políticas para a promoção da eqüidade em saúde e mobilizar setores da sociedade para o debate e posicionamento em torno dos DSS e do enfrentamento das iniqüidades de saúde.

Palavras-chave: Determinantes sociais; eqüidade; políticas públicas; promoção da saúde; comissão nacional.

Recebido em: 28/02/2007.

Aprovado em: 15/03/2007. 


\section{Que se entende por determinantes sociais da saúde?}

As diversas definições de determinantes sociais de saúde (DSS) expressam, com maior ou menor nível de detalhe, o conceito atualmente bastante generalizado de que as condições de vida e trabalho dos indivíduos e de grupos da população estão relacionadas com sua situação de saúde. Para a Comissão Nacional sobre os Determinantes Sociais da Saúde (CNDSS), os DSS são os fatores sociais, econômicos, culturais, étnicos/raciais, psicológicos e comportamentais que influenciam a ocorrência de problemas de saúde e seus fatores de risco na população. A comissão homônima da Organização Mundial da Saúde (OMS) adota uma definição mais curta, segundo a qual os DSS são as condições sociais em que as pessoas vivem e trabalham. Nancy Krieger (2001) introduz um elemento de intervenção, ao defini-los como os fatores e mecanismos através dos quais as condições sociais afetam a saúde e que potencialmente podem ser alterados através de ações baseadas em informação. Tarlov (1996) propõe, finalmente, uma definição bastante sintética, ao entendêlos como as características sociais dentro das quais a vida transcorre.

Embora, como já mencionado, tenha-se hoje alcançado certo consenso sobre a importância dos DSS na situação de saúde, esse consenso foi sendo construído ao longo da história. Entre os diversos paradigmas explicativos para os problemas de saúde, em meados do século XIX predominava a teoria miasmática, que conseguia responder às importantes mudanças sociais e práticas de saúde observadas no âmbito dos novos processos de urbanização e industrialização ocorridos naquele momento histórico. Estudos sobre a contaminação da água e dos alimentos, assim como sobre riscos ocupacionais, trouxeram importante reforço para o conceito de miasma e para as ações de saúde pública (SUSSER, 1998).

Virchow, um dos mais destacados cientistas vinculados a essa teoria, entendia que a "ciência médica é intrínseca e essencialmente uma ciência social", que as condições econômicas e sociais exercem um efeito importante sobre a saúde e a doença e que tais relações devem ser submetidas à pesquisa científica. Entendia também que o próprio termo "saúde pública" expressa seu caráter político e que sua prática implica necessariamente a intervenção na vida política e social para identificar e eliminar os fatores que prejudicam a saúde da população (ROSEN, 1980). Outros autores que merecem destaque nessa corrente de pensamento são Chadwick, com seu Report on the sanitary condition of the labouring population of Great Britain, de 1842, Villermé, com Tableau de 
l'état physique et moral des ouvriers de Paris, de 1840, e Engels, com A situação das classes trabalhadoras na Inglaterra, Londres, de 1845.

Nas últimas décadas do século XIX, com o extraordinário trabalho de bacteriologistas como Koch e Pasteur, afirma-se um novo paradigma para a explicação do processo saúde-doença. A história da criação da primeira escola de saúde pública nos Estados Unidos, na Universidade Johns Hopkins, é um interessante exemplo do processo de afirmação da hegemonia desse "paradigma bacteriológico". Desde 1913, quando a Fundação Rockefeller decide propor o estabelecimento de uma escola para treinar os profissionais de saúde pública, até a decisão, em 1916, de financiar sua implantação em Johns Hopkins, há um importante debate entre diversas correntes e concepções sobre a estruturação do campo da saúde pública. No centro do debate estiveram questões como: deve a saúde pública tratar do estudo de doenças específicas, como um ramo especializado da medicina, baseando-se fundamentalmente na microbiologia e nos sucessos da teoria dos germes ou deve centrar-se no estudo da influência das condições sociais, econômicas e ambientais na saúde dos indivíduos? Outras questões relacionadas: a saúde e a doença devem ser pesquisadas no laboratório, com o estudo biológico dos organismos infecciosos, ou nas casas, nas fábricas e nos campos, buscando conhecer as condições de vida e os hábitos de seus hospedeiros?

Como se pode ver, o conflito entre saúde pública e medicina e entre os enfoques biológico e social do processo saúde-doença estiveram no centro do debate sobre a configuração desse novo campo de conhecimento, de prática e de educação. Ao final desse processo, Hopkins foi escolhida pela excelência de sua escola de medicina, de seu hospital e de seu corpo de pesquisadores médicos. Esta decisão representou o predomínio do conceito da saúde pública orientada ao controle de doenças específicas, fundamentada no conhecimento científico baseado na bacteriologia e contribuiu para "estreitar" o foco da saúde pública, que passa a distanciar-se das questões políticas e dos esforços por reformas sociais e sanitárias de caráter mais amplo. A influência desse processo e do modelo por ele gerado não se limita à escola de saúde pública de Hopkins, estendendo-se por todo o país e internacionalmente. O modelo serviu para que nos anos seguintes a Fundação Rockefeller apoiasse o estabelecimento de escolas de saúde pública no Brasil (Faculdade de Higiene e Saúde Pública de São Paulo), Bulgária, Canadá, Checoslováquia, Inglaterra, Hungria, Índia, Itália, Japão, Noruega, Filipinas, Polônia, Romênia, Suécia, Turquia e Iugoslávia (FEE, 1987). 
Apesar da preponderância do enfoque médico biológico na conformação inicial da saúde pública como campo científico, em detrimento dos enfoques sociopolíticos e ambientais, observa-se, ao longo do século XX, uma permanente tensão entre essas diversas abordagens. A própria história da OMS oferece interessantes exemplos dessa tensão, observando-se períodos de forte preponderância de enfoques mais centrados em aspectos biológicos, individuais e tecnológicos, intercalados com outros em que se destacam fatores sociais e ambientais. A definição de saúde como um estado de completo bem-estar físico, mental e social, e não meramente a ausência de doença ou enfermidade, inserida na Constituição da OMS no momento de sua fundação, em 1948, é uma clara expressão de uma concepção bastante ampla da saúde, para além de um enfoque centrado na doença. Entretanto, na década de 50, com o sucesso da erradicação da varíola, há uma ênfase nas campanhas de combate a doenças específicas, com a aplicação de tecnologias de prevenção ou cura.

A Conferência de Alma-Ata, no final dos anos 70, e as atividades inspiradas no lema "Saúde para todos no ano 2000" recolocam em destaque o tema dos determinantes sociais. Na década de 80 , o predomínio do enfoque da saúde como um bem privado desloca novamente o pêndulo para uma concepção centrada na assistência médica individual, a qual, na década seguinte, com o debate sobre as Metas do Milênio, novamente dá lugar a uma ênfase nos determinantes sociais que se afirma com a criação da Comissão sobre Determinantes Sociais da Saúde da OMS, em 2005.

\section{O estudo dos determinantes sociais da saúde}

Nas últimas décadas, tanto na literatura nacional, como internacional, observa-se um extraordinário avanço no estudo das relações entre a maneira como se organiza e se desenvolve uma determinada sociedade e a situação de saúde de sua população (ALMEIDA-FILHO, 2002). Esse avanço é particularmente marcante no estudo das iniqüidades em saúde, ou seja, daquelas desigualdades de saúde entre grupos populacionais que, além de sistemáticas e relevantes, são também evitáveis, injustas e desnecessárias (WHITEHEAD, 2000). Segundo Nancy Adler (2006), podemos identificar três gerações de estudos sobre as iniqüidades em saúde. A primeira geração se dedicou a descrever as relações entre pobreza e saúde; a segunda, a descrever os gradientes de saúde de acordo com vários critérios de estratificação socioeconômica; e a terceira e atual geração está dedicada principalmente aos 
estudos dos mecanismos de produção das iniqüidades ou, para usar a expressão de Adler, está dedicada a responder à pergunta: como a estratificação econômico-social consegue "entrar" no corpo humano?

O principal desafio dos estudos sobre as relações entre determinantes sociais e saúde consiste em estabelecer uma hierarquia de determinações entre os fatores mais gerais de natureza social, econômica, política e as mediações através das quais esses fatores incidem sobre a situação de saúde de grupos e pessoas, já que a relação de determinação não é uma simples relação direta de causa-efeito. É através do conhecimento deste complexo de mediações que se pode entender, por exemplo, por que não há uma correlação constante entre os macroindicadores de riqueza de uma sociedade, como o PIB, com os indicadores de saúde. Embora o volume de riqueza gerado por uma sociedade seja um elemento fundamental para viabilizar melhores condições de vida e de saúde, o estudo dessas mediações permite entender por que existem países com um PIB total ou PIB per capita muito superior a outros que, no entanto, possuem indicadores de saúde muito mais satisfatórios. O estudo dessa cadeia de mediações permite também identificar onde e como devem ser feitas as intervenções, com o objetivo de reduzir as iniqüidades de saúde, ou seja, os pontos mais sensíveis onde tais intervenções podem provocar maior impacto.

Outro desafio importante em termos conceituais e metodológicos se refere à distinção entre os determinantes de saúde dos indivíduos e os de grupos e populações, pois alguns fatores que são importantes para explicar as diferenças no estado de saúde dos indivíduos não explicam as diferenças entre grupos de uma sociedade ou entre sociedades diversas. Em outras palavras, não basta somar os determinantes de saúde identificados em estudos com indivíduos para conhecer os determinantes de saúde no nível da sociedade. As importantes diferenças de mortalidade constatadas entre classes sociais ou grupos ocupacionais não podem ser explicadas pelos mesmos fatores aos quais se atribuem as diferenças entre indivíduos, pois se controlamos esses fatores (hábito de fumar, dieta, sedentarismo etc.), as diferenças entre estes estratos sociais permanecem quase inalteradas.

Enquanto os fatores individuais são importantes para identificar que indivíduos no interior de um grupo estão submetidos a maior risco, as diferenças nos níveis de saúde entre grupos e países estão mais relacionadas com outros fatores, principalmente o grau de eqüidade na distribuição de renda. Por exemplo, o Japão é o país com a maior expectativa de vida ao nascer, não porque os 
japoneses fumam menos ou fazem mais exercícios, mas porque o Japão é um dos países mais igualitários do mundo. Ao confundir os níveis de análise e tratar de explicar a saúde das populações a partir de resultados de estudos realizados com indivíduos, estaríamos aceitando o contrário da chamada "falácia ecológica" (KAWACHI et al., 1997; WILKINSON, 1997; PELEGRINI FILHO, 2000).

O clássico estudo de Rose e Marmot (1981) sobre a mortalidade por doença coronariana em funcionários públicos ingleses ilustra muito bem esta situação. Fixando como um o risco relativo de morrer por esta doença no grupo ocupacional de mais alto nível na hierarquia funcional, os funcionários de níveis hierárquicos inferiores, como profissional/executivo, atendentes e outros, teriam risco relativo aproximadamente duas, três e quatro vezes maiores, respectivamente. Os autores encontraram que os fatores de risco individuais, como colesterol, hábito de fumar, hipertensão arterial e outros explicavam apenas 35 a $40 \%$ da diferença, sendo que os restantes $60-65 \%$ estavam basicamente relacionados aos DSS.

Há várias abordagens para o estudo dos mecanismos através dos quais os DSS provocam as iniqüidades de saúde. A primeira delas privilegia os "aspectos físico-materiais" na produção da saúde e da doença, entendendo que as diferenças de renda influenciam a saúde pela escassez de recursos dos indivíduos e pela ausência de investimentos em infra-estrutura comunitária (educação, transporte, saneamento, habitação, serviços de saúde etc.), decorrentes de processos econômicos e de decisões políticas. Outro enfoque privilegia os "fatores psicosociais", explorando as relações entre percepções de desigualdades sociais, mecanismos psicobiológicos e situação de saúde, com base no conceito de que as percepções e as experiências de pessoas em sociedades desiguais provocam estresse e prejuízos à saúde. Os enfoques "ecossociais" e os chamados "enfoques multiníveis" buscam integrar as abordagens individuais e grupais, sociais e biológicas numa perspectiva dinâmica, histórica e ecológica.

Finalmente, há os enfoques que buscam analisar as relações entre a saúde das populações, as desigualdades nas condições de vida e o grau de desenvolvimento da trama de vínculos e associações entre indivíduos e grupos. Esses estudos identificam o desgaste do chamado "capital social", ou seja, das relações de solidariedade e confiança entre pessoas e grupos, como um importante mecanismo através do qual as iniqüidades de renda impactam negativamente a situação de saúde. Países com frágeis laços de coesão social, 
ocasionados pelas iniqüidades de renda, são os que menos investem em capital humano e em redes de apoio social, fundamentais para a promoção e proteção da saúde individual e coletiva. Esses estudos também procuram mostrar por que não são as sociedades mais ricas as que possuem melhores níveis de saúde, mas as que são mais igualitárias e com alta coesão social.

Diversos são os modelos que procuram esquematizar a trama de relações entre os diversos fatores estudados através desses diversos enfoques. Dois modelos serão analisados a seguir: o modelo de Dahlgren e Whitehead (GUNNING-SCHEPERS, 1999) e o modelo de Didericksen e outros (EVANS et al., 2001).

O modelo de Dahlgren e Whitehead inclui os DSS dispostos em diferentes camadas, desde uma camada mais próxima dos determinantes individuais até uma camada distal, onde se situam os macrodeterminantes. Apesar da facilidade da visualização gráfica dos DSS e sua distribuição em camadas, segundo seu nível de abrangência, o modelo não pretende explicar com detalhes as relações e mediações entre os diversos níveis e a gênese das iniqüidades. Como se pode ver na figura 1, os indivíduos estão na base do modelo, com suas características individuais de idade, sexo e fatores genéticos que, evidentemente, exercem influência sobre seu potencial e suas condições de saúde. Na camada imediatamente externa aparecem o comportamento e os estilos de vida individuais. Esta camada está situada no limiar entre os fatores individuais e os DSS, já que os comportamentos, muitas vezes entendidos apenas como de responsabilidade individual, dependentes de opções feitas pelo livre arbítrio das pessoas, na realidade podem também ser considerados parte dos DSS, já que essas opções estão fortemente condicionadas por determinantes sociais - como informações, propaganda, pressão dos pares, possibilidades de acesso a alimentos saudáveis e espaços de lazer etc. 


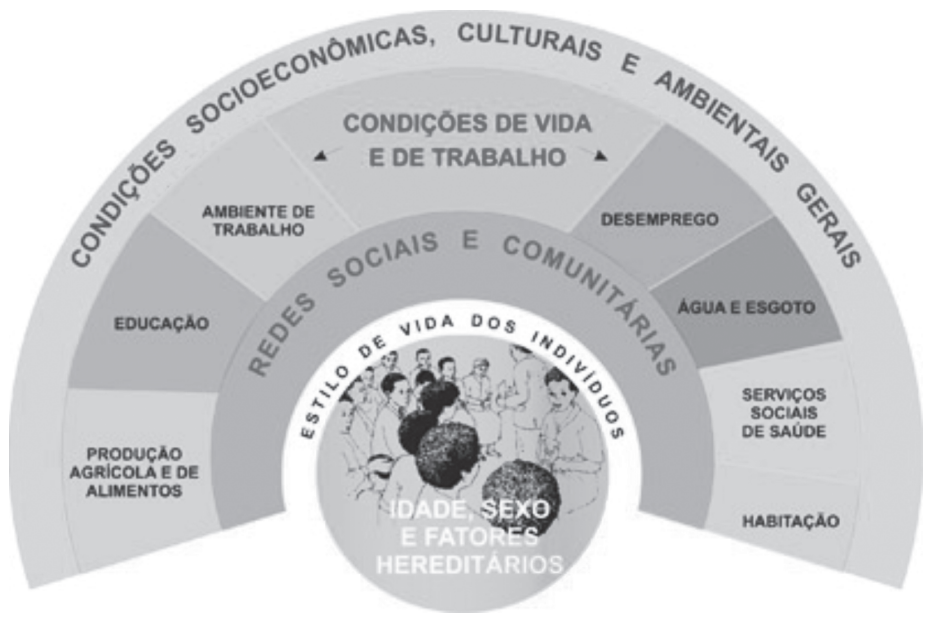

Figura 1 - Determinantes sociais: modelo de Dahlgren e Whitehead

A camada seguinte destaca a influência das redes comunitárias e de apoio, cuja maior ou menor riqueza expressa o nível de coesão social que, como vimos, é de fundamental importância para a saúde da sociedade como um todo. No próximo nível estão representados os fatores relacionados a condições de vida e de trabalho, disponibilidade de alimentos e acesso a ambientes e serviços essenciais, como saúde e educação, indicando que as pessoas em desvantagem social correm um risco diferenciado, criado por condições habitacionais mais humildes, exposição a condições mais perigosas ou estressantes de trabalho e acesso menor aos serviços. Finalmente, no último nível estão situados os macrodeterminantes relacionados às condições econômicas, culturais e ambientais da sociedade e que possuem grande influência sobre as demais camadas.

Necessário mencionar, pela crescente influência sobre as condições sociais, econômicas e culturais dos países, o fenômeno da globalização. Suas principais características, assim como a influência da globalização sobre a pobreza e as condições de saúde, e sobre as condições de vida em geral foram analisadas por Buss (2006). 
O modelo de Diderichsen e Hallqvist, de 1998, foi adaptado por Diderichsen, Evans e Whitehead (2001). Esse modelo enfatiza a estratificação social gerada pelo contexto social, que confere aos indivíduos posições sociais distintas, as quais por sua vez provocam diferenciais de saúde. No diagrama abaixo (figura 2), (I) representa o processo segundo o qual cada indivíduo ocupa determinada posição social como resultado de diversos mecanismos sociais, como o sistema educacional e o mercado de trabalho. De acordo com a posição social ocupada pelos diferentes indivíduos, aparecem diferenciais, como o de exposição a riscos que causam danos à saúde (II); o diferencial de vulnerabilidade à ocorrência de doença, uma vez exposto a estes riscos (III); e o diferencial de conseqüências sociais ou físicas, uma vez contraída a doença (IV). Por "conseqüências sociais" entende-se o impacto que a doença pode ter sobre a situação socioeconômica do indivíduo e sua família.

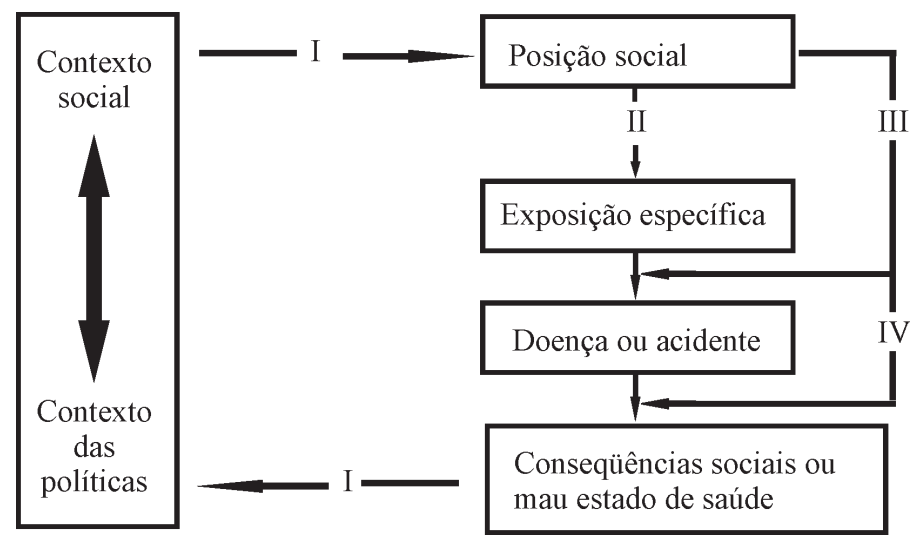

Figura 2 - Determinantes sociais: modelo de Diderichsen e Hallqvist

\section{As intervenções sobre os determinantes sociais da saúde}

O modelo de Dahlgren e Whitehead e o de Diderichsen permitem identificar pontos para intervenções de políticas, no sentido de minimizar os diferenciais de DSS originados pela posição social dos indivíduos e grupos.

Tomando o modelo de camadas de Dahlgren e Whitehead, o primeiro nível relacionado aos fatores comportamentais e de estilos de vida indica que 
estes estão fortemente influenciados pelos DSS, pois é muito difícil mudar comportamentos de risco sem mudar as normas culturais que os influenciam. Atuando-se exclusivamente sobre os indivíduos, às vezes se consegue que alguns deles mudem de comportamento, mas logo eles serão substituídos por outros (ROSE, 1992). Para atuar nesse nível de maneira eficaz, são necessárias políticas de abrangência populacional que promovam mudanças de comportamento, através de programas educativos, comunicação social, acesso facilitado a alimentos saudáveis, criação de espaços públicos para a prática de esportes e exercícios físicos, bem como proibição à propaganda do tabaco e do álcool em todas as suas formas.

O segundo nível corresponde às comunidades e suas redes de relações. Como já mencionado, os laços de coesão social e as relações de solidariedade e confiança entre pessoas e grupos são fundamentais para a promoção e proteção da saúde individual e coletiva. Aqui se incluem políticas que busquem estabelecer redes de apoio e fortalecer a organização e participação das pessoas e das comunidades, especialmente dos grupos vulneráveis, em ações coletivas para a melhoria de suas condições de saúde e bem-estar, e para que se constituam em atores sociais e participantes ativos das decisões da vida social.

O terceiro nível se refere à atuação das políticas sobre as condições materiais e psicossociais nas quais as pessoas vivem e trabalham, buscando assegurar melhor acesso à água limpa, esgoto, habitação adequada, alimentos saudáveis e nutritivos, emprego seguro e realizador, ambientes de trabalho saudáveis, serviços de saúde e de educação de qualidade e outros. Em geral essas políticas são responsabilidade de setores distintos, que freqüentemente operam de maneira independente, obrigando o estabelecimento de mecanismos que permitam uma ação integrada.

O quarto nível de atuação se refere à atuação ao nível dos macrodeterminantes, através de políticas macroeconômicas e de mercado de trabalho, de proteção ambiental e de promoção de uma cultura de paz e solidariedade que visem a promover um desenvolvimento sustentável, reduzindo as desigualdades sociais e econômicas, as violências, a degradação ambiental e seus efeitos sobre a sociedade (CNDSS, 2006; PELEGRINI FILHO, 2006).

O outro modelo, proposto por Diderichsen et al., permite também identificar alguns pontos de incidência de políticas que atuem sobre os mecanismos de estratificação social e sobre os diferenciais de exposição, de vulnerabilidade e de suas conseqüências. 
Embora a intervenção sobre os mecanismos de estratificação social seja de responsabilidade de outros setores, ela é das mais cruciais para combater as iniqüidades de saúde. Aqui se incluem políticas que diminuam as diferenças sociais, como as relacionadas ao mercado de trabalho, educação e seguridade social, além de um sistemático acompanhamento de políticas econômicas e sociais para avaliar seu impacto e diminuir seus efeitos sobre a estratificação social.

O segundo conjunto de políticas busca diminuir os diferenciais de exposição a riscos, tendo como alvo, por exemplo, os grupos que vivem em condições de habitação insalubres, trabalham em ambientes pouco seguros ou estão expostos a deficiências nutricionais. Aqui se incluem também políticas de fortalecimento de redes de apoio a grupos vulneráveis para mitigar os efeitos de condições materiais e psicossociais adversas. Quanto ao enfrentamento dos diferenciais de vulnerabilidade, são mais efetivas as intervenções que buscam fortalecer a resistência a diversas exposições, como por exemplo, a educação das mulheres para diminuir sua própria vulnerabilidade e a de seus filhos. A intervenção no sistema de saúde busca reduzir os diferenciais de conseqüências ocasionadas pela doença, aqui incluindo a melhoria da qualidade dos serviços a toda a população, apoio a deficientes, acesso a cuidados de reabilitação e mecanismos de financiamento eqüitativos, que impeçam o empobrecimento adicional causado pela doença.

Essas intervenções sobre níveis macro, intermediário ou micro de DSS, com vistas a diminuir as iniqüidades relacionadas à estratificação social, além de obrigarem a uma atuação coordenada intersetorial abarcando diversos níveis da administração pública, devem estar também acompanhadas por políticas mais gerais de caráter transversal que busquem fortalecer a coesão e ampliar o "capital social" das comunidades vulneráveis, e promover a participação social no desenho e implementação de políticas e programas (CSDH, 2006).

A evolução conceitual e prática do movimento de promoção da saúde em nível mundial indica uma ênfase cada vez maior na atuação sobre os DSS, constituindo importante apoio para a implantação das políticas e intervenções acima mencionadas.

\section{A Comissão Nacional sobre os Determinantes Sociais da Saúde (CNDSS)}

O conhecimento e as intervenções sobre os DSS no Brasil deverão receber importante impulso, com a criação da Comissão Nacional sobre 
Determinantes Sociais da Saúde (CNDSS). Essa Comissão foi estabelecida em 13 de março de 2006, através de Decreto Presidencial, com um mandato de dois anos. A criação da CNDSS é uma resposta ao movimento global em torno dos DSS desencadeado pela OMS, que em março de 2005 criou a Comissão sobre Determinantes Sociais da Saúde (Commission on Social Determinants of Health - CSDH), com o objetivo de promover, em âmbito internacional, uma tomada de consciência sobre a importância dos determinantes sociais na situação de saúde de indivíduos e populações e sobre a necessidade do combate às iniqüidades de saúde por eles geradas.

A CNDSS está integrada por 16 personalidades expressivas de nossa vida social, cultural, científica e empresarial. ${ }^{1}$ Sua constituição diversificada é uma expressão do reconhecimento de que a saúde é um bem público, construído com a participação solidária de todos os setores da sociedade brasileira. O Decreto Presidencial que criou a CNDSS constituiu também um Grupo de Trabalho Intersetorial, integrado por diversos ministérios relacionados com os DSS, além dos Conselhos Nacionais de Secretários Estaduais e Municipais de Saúde (CONASS e CONASEMS). O trabalho articulado da CNDSS com esse Grupo permite que se multipliquem ações integradas entre as diversas esferas da administração pública, e que as já existentes ganhem maior coerência e efetividade.

As atividades da CNDSS têm como referência o conceito de saúde, tal como a concebe a OMS - "um estado de completo bem-estar físico, mental e social e não meramente a ausência de doença ou enfermidade" - e o preceito constitucional de reconhecer a saúde como um "direito de todos e dever do Estado, garantido mediante políticas sociais e econômicas que visem à redução do risco de doença e outros agravos e ao acesso universal e igualitário às ações e serviços para sua promoção, proteção e recuperação" (artigo 196 da Constituição brasileira de 1988).

Três compromissos vêm orientando a atuação da Comissão:

- Compromisso com a ação: implica apresentar recomendações concretas de políticas, programas e intervenções para o combate às iniqüidades de saúde geradas pelos DSS.

- Compromisso com a eqüidade: a promoção da eqüidade em saúde é fundamentalmente um compromisso ético e uma posição política que orienta as ações da CNDSS para assegurar o direito universal à saúde. 
- Compromisso com a evidência: as recomendações da Comissão devem estar solidamente fundamentadas em evidências científicas, que permitam, por um lado, entender como operam os determinantes sociais na geração das iniqüidades em saúde e, por outro, como e onde devem incidir as intervenções para combatê-las e que resultados podem ser esperados em termos de efetividade e eficiência.

Os principais objetivos da CNDSS são:

- produzir conhecimentos e informações sobre os DSS no Brasil;

- apoiar o desenvolvimento de políticas e programas para a promoção da eqüidade em saúde;

- promover atividades de mobilização da sociedade civil para tomada de consciência e atuação sobre os DSS.

Para o alcance desses objetivos, a CNDSS vem desenvolvendo as seguintes linhas de atuação:

1) Produção de conhecimentos e informações sobre as relações entre os determinantes sociais e a situação de saúde, particularmente as iniqüidades de saúde, com vistas a fundamentar políticas e programas. No âmbito desta linha de atuação, a CNDSS, o Departamento de Ciência e Tecnologia do Ministério da Saúde e o CNPq lançaram um edital de pesquisa que permitiu apoiar projetos de pesquisa sobre DSS por um montante de cerca de quatro milhões de reais. Os pesquisadores responsáveis por esses projetos e gestores locais e estaduais convidados estão conformando uma rede de colaboração e intercâmbio para seguimento dos projetos e discussão de implicações para políticas de seus resultados intermediários. Ainda no âmbito desta linha de atuação, foram identificados e avaliados sistemas de informação de abrangência nacional sobre DSS e foi realizado um seminário internacional sobre metodologias de avaliação de intervenções sobre os DSS. Os resultados dessas atividades estarão em breve disponíveis no site da CNDSS.

2) Promoção, apoio, seguimento e avaliação de políticas, programas e intervenções governamentais e não-governamentais realizadas em nível local, regional e nacional. O GT Intersetorial deve constituir o principal instrumento para o desenvolvimento desta linha de atuação.

3) Desenvolvimento de ações de promoção e mobilização junto a diversos setores da sociedade civil, para a tomada de consciência sobre a 
importância das relações entre saúde e condições de vida e sobre as possibilidades de atuação para diminuição das iniqüidades de saúde. Membros da CNDSS e da secretaria técnica vêm participando de congressos e reuniões nacionais e internacionais e utilizando meios de comunicação de massa para o desenvolvimento desta linha de atuação. Em breve será organizado um fórum de discussão nacional e regional, com a participação de organizações nãogovernamentais que atuam em áreas relacionadas com os DSS.

4) Portal sobre DSS: a CNDSS mantém uma página institucional (www.determinates.fiocruz.br) com informações sobre as atividades que vem desenvolvendo, além de publicações de interesse. Em breve será lançado um Portal sobre DSS, onde, além de informações sobre as atividades da CNDSS, serão incluídos dados, informações e conhecimentos sobre DSS existentes nos sistemas de informação e na literatura mundial e nacional. Esse portal deve também se constituir num espaço de interação para intercâmbio e discussão de grupos estratégicos relacionados aos DSS, como pesquisadores, tomadores de decisão, profissionais de comunicação e outros.

A partir do segundo semestre de 2007, a CNDSS começará a publicar seu relatório final em fascículos, para prestar contas sobre o cumprimento de seus objetivos, traçar um panorama geral da situação de saúde do país e propor políticas e programas relacionados aos DSS. Estamos convencidos de que as atividades da CNDSS e seus desdobramentos futuros serão uma valiosa contribuição para o avanço do processo de reforma sanitária brasileira e para a construção de uma sociedade mais humana e justa.

\section{Referências}

ADLER, N. Behavioral and social sciences research contributions in NIH Conference on Understanding and Reducing Disparities in Health, October 2324, 2006 NIH Campus, Bethesda, Maryland. Disponível em: http:// obssr.od.nih.gov/HealthDisparities/presentation.html. Acesso em: 14/02/07.

ALMEIDA FILHO, N. et al. Research on health inequalities in Latin America and the Caribbean: Bibliometric analysis (1971-2000) and descriptive content analysis (1971-1995). Am J Public Health, n. 93, p. 2.037-2.043, 2003.

BUSS, P. M. Globalização, pobreza e saúde. Conferência Leavell apresentada ao VIII Congresso Brasileiro de Saúde Coletiva e XI Congresso Mundial de 
Saúde Pública, Rio de Janeiro, agosto de 2006. Disponível em: www.fíocruz.br. Acesso em: 14/02/07.

COMISSÃO NACIONAL SOBRE OS DETERMINANTES SOCIAIS DA SAÚDE (CNDSS). Carta aberta aos candidatos à Presidência da República. Setembro de 2006. Disponível em: www.determinantes.fiocruz.br. Acesso em: 15/02/07.

COMMISSION ON SOCIAL DETERMINANTS OF HEALTH (CSDH). A conceptual framework for action on social determinants of health. Disponível em www.determinantes.fiocruz.br. Acesso em: 10/02/07.

EVANS, T. et al. Challenging inequities in health from ethics to action. Oxford: Oxford University Press, 2001.

FEE, E. Disease and discovery: a history of the Johns Hopkins School of Hygiene and Public Health. Baltimore :The Johns Hopkins University Press, 1987.

GUNNING-SCHEPERS, L. J. Models: instruments for evidence based policy. J Epidemiology Community Health, n. 53, p. 263, 1999.

KAWACHI, I. et al. Social capital, income inequality and mortality. Am J Public Health, n. 87, p. 1.491-1.498, 1997.

KRIEGER N. A Glossary for social epidemiology. J. Epidemiology Community Health, n. 55, p. 693-700, 2001.

PELLEGRINI FILHO A. Compromisso com a ação. Radis, n. 47, p.12-14, jul. 2006.

PELLEGRINI FILHO, A. Ciencia en pro de la Salud. Publicación científica y técnica $\mathrm{n}^{0}$.578. Washington DC: OPS/OMS, 2000.

ROSE, G. The strategy of preventive medicine. Oxford: Oxford University Press, 1992.

ROSE, G.; MARMOT, M. Social class and coronary heart disease. British Heart Journal, p. 13-19, 1981.

ROSEN, G. Da polícia médica à medicina social. Rio de Janeiro, Graal, 1980.

SUSSER, M. Does risk factor epidemiology put epidemiology at risk? Peering into the future. J Epidemiol Community Health, n. 52, p. 608-611, 1998.

TARLOV, A. Social Determinants of Health: the sociobiological translation. In: 
BLANE, D.; BRUNNER,E.; WILKINSON, R. (Eds.). Health and Social Organization. London: Routledge. p. 71-93, 1996.

WHITEHEAD, M. The concepts and principles of equity and health. EUR/ ICP/RPD 414, 7734r, Geneva: WHO, 2000.

WILKINSON, R. Unhealthy societies. New York: Routledge, 1997.

\section{NOTAS}

- Presidente da Fundação Oswaldo Cruz (FIOCRUZ), Membro titular da Academia Nacional de Medicina e coordenador da Comissão Nacional sobre Determinantes Sociais da Saúde (CNDSS). Endereço eletrônico: buss@ fiocruz.br.

- Pesquisador titular da FIOCRUZ e coordenador da Secretaria Técnica da CNDSS. Endereço eletrônico: pellegrini@fiocruz.br.

${ }^{1}$ São membros da CNDSS: Adib Jatene, Aloysio Teixeira, César Victora, Dalmo Dallari, Eduardo Eugênio Gouveia Vieira, Elza Berquó, Jaguar, Jairnilson Paim, Lucélia Santos, Moacyr Scliar, Roberto Esmeraldi, Rubem César Fernandes, Sandra de Sá, Sonia Fleury, Zilda Arns e Paulo M. Buss (coordenador). 


\section{Health and its Social Determinants}

This article aims to analyze the relationships between health and its social determinants, initially presenting the concept of Social Determinants of Health (SDH) and the historical evolution of paradigms that account for the health/disease process at societal level since mid-1800's. The main advances and challenges in the study of SDH are presented with emphasis on the new approaches and frameworks to explain the complex relationships between the various levels of SDH and health outcomes. Based on these frameworks, some entry points for interventions on SDH and types of policies and programs to tackle health inequities are explored. Finally, the article presents the objectives, lines of actions and main activities developed by the National Commission on Social Determinants of Health, created in March 2005 to promote studies on SDH, to recommend policies for promotion of health equity and to mobilize sectors in society to promote awareness about the importance of SDH and the fight against health inequities.

Key words: Social determinants; equity; public policies; health promotion; national commission. 